\title{
Sull' azione dell' elettricità nello sviluppo delle uova degli Anfibi.
}

\author{
[Istituto Anatomico di Firenze diretto dal Prof. G. Chiarugr.]
}

Ricerche sperimentali

del

Dott. Umberto Rossi,

Libero docento e Io $\Lambda$ iuto.

Con tav. IX-XIV e 1 figura nel testo.

Eingegangen am 15. Juni 1896.

Parte storica e scopo delle ricerche.

Il primo a sperimentare l'azione dell' elettricità sullo sviluppo delle uova di vertebrati fu Rusconi (1). Facendo agire correnti generate da una pila di VoLTA sulle ova di Rana artificialmente fecondate, ossservò che si sviluppavano un pó più presto di quelle non galvanizzate. A distanza di parecchi anni seguono le ricerche di Lombardini (2) il quale studiò l'azione dell' elettricità sugli $A n-$ $f b i$ e sngli Uccelli. Riguardo ai primi questo autore si servi nei suoi esperimenti di un' elemento Bunsen alto 12 centimetri apparecchiato con acqua e poche gocce di acido solforico e di una pila composta con lamine di zinco e rame immerse nell' acqua salata. Con questi mezzi vide che eletrizzazioni di breve durata (40 minuti) acceleravano lo sviluppo, mentre nova di Rana sottoposte a prolungata azione della corrente ( 8 giorni) presentavano al termine dell'esperimento segni manifesti di alterazione. Sorpreso da certe apparenze esteriori tentò LoMbardiñ di studiare anche le possibili modificazioni di struttura delle.ova e a tale scopo ne schiacciò alcune tra due vetrini e sottopostole all' esame microscopico notò numerose varietà di forma delle sferule vitelline. In seguito a tali risultati 
coucluse che l'azione dell' elettricità quando è di breve durata e non molto forte, oppure continua ma debolissima, non interompe assolutamente lo sviluppo delle nova di Rana, ma che tale azione accelerando i moti embriogenici spesso li disordina. Per ciò che riguarda gli Uccelli Lombardini ha studiato gli effetti dell' elettricita sulle parti elementari dell' uovo e sui tessuti embrionali, e cioè lo stato degli organi embrionali, lo stato molecolare delle materie nutritizic e la loro composizione chimica dopo l'azione dell' elettricití. Le anomalie principali da esso riscontrate si riassumouo in connessioni irregolari delle ossa, comessioni irregolari del cuore (ectopie), divisione dell' allantoide, sviluppo patologico della tosta e del cuore, come protuberanza irregolare dell' encefalo, ipersarcosi del cuore, iperemia della testa con adesione dell amnios a questa parte, tumori vaseolari degli occhi, lacerazioni delle pareti del cranio, disuguaglianza delle vescicole cerebrali, tumori dell' encefalo e del cranio. Oltre a cio condizioni patologiche generali quali idroemia e deformazioni di tutto il corpo.

Fasola (3) studia nel 1857 nel laboratorio di Duval l'azione di deboli correnti indotte sullo sviluppo delle nova di Rana. Così conclude l'antore: „Se con determinate norme e con le dorute precauzioni si fanno agire delle deboli correnti indotte sulle uova di Rana durante i primi giorni del loro sviluppo, si ha per effetto di accelerare alquanto lo sviluppo stesso e quindi di anticipare di alcun poco la comparsa delle larye ..... $\dot{\mathrm{E}}$ possibile, continua, che quell' elettriciti agisea in quanto si trasforma in calore per la resistenza incontrata nollo strato di mucina e negli elementi del vitello; è possibile che modificando lo stato di equilibrio delle molecole, essa aumenti le affinita elettive di determinati elementi chimici e ne renda quindi più pronte e più attive le azioni reciproche; è possibile in fine, specialmente dal punto di vista di ipotesi ogrgi abbastanza accreditate, che essa faciliti particolari orientazioni molecolari aventi forse una parte ragguardevole in tutti i fenomeni organici. Certo è che una influenza acceleratrice esiste e si manifesta chiaramente purchè le esperienze rengano escguite con le dovute norme e cautele e l'averlo constatato viene se non altro a rendere ancora più probabile l'ipotesi non ancora dimostrata, ma snffragata già da numerosissimi fatti, che l'elettriciti e il magnetismo abbiano uwa funzione importantissima, forse capitale, anche nei fenomeni piu intimi e delicati della vita. „FASOLA si domanda poi se l'accelerazione del processo evolutivo sia di danno o no ai nuovi esseri. Stando al 
fatto che gli sviluppi affrettati danno quasi sempre organismi deboli, male costituiti ed anche mostruosi e che ebbe molte larve morte nei saggi che presentarono la maggiore precocità, crede che l'accelerazione riesca dannosa.

WINDLe (4) nel 1893 ripete le ricerche di LoMbardini e di Prevost e Dumas $\left.(5)^{1}\right)$ e nota pure esso negli embrioni di pollo malformazioni dell' area vascolosa, mancanza di un' occhio, disuguaglianza di volume fra il destro e il sinistro. Nel 1895 (6) l'autore continna i suoi esperimenti intorno all' influenza della elettricità generata da pile Davré sullo sviluppo delle uova di Trota e conclude che l'elettricità produce un' arresto di sviluppo.

Dareste (7) pure nel 1895 si occupa di studiare l'influenza dell' elettricità sull' evoluzione dell' embrione di pollo. Ecco come ha proceduto nei suoi esperimenti.

$1^{\circ} \mathrm{Ha}$ collocato nova di pollo per un' ora in un solenoide di Tesla. ,

$2^{\circ}$ Ha sottomesso le uova all' azione di scintille prodotte dalla macchina BoNETTX munita dei suoi condensatori.

$3^{0} \mathrm{Ha}$ sottomesso le uova all' azione di scintille di induzione prodotte dalla bobina di RHUMkoRfF per 1-3 minuti; la corrente entrava o per il p. +0 per il p. - Dopo l'eletrizzazione le uova venivano collocate in una incubatrice a $38^{\circ}$ circa e aperte dopo 4-8 giorni. I risultati sono i seguenti: Le uova del solenoide si svilupparono con leggero ritardo in qualcuna, ma normalmente. Quelle delle altre due serie si svilupparono, pochissime normalmente, molte anormalmente, ma secondo i tipi teratologici osservati nelle esperienze precedenti, essendo la gravità dell' anomalia in rapporto con l'intensità dell' azione teratogenica. Secondo DARESTe l'elettricità agisce come tutte le cause che modificano l'evoluzione e imprimono ed essa una direzione differente dalla direzione normale.

Sorvolando sulle esperienze di Verson e QUAJaT (8) di BeLlati

1) A proposito delle ricerche di Prevost e Dumas poco conosciamo. DaRESTE nelle sue $\gg$ Recherches sur la production artificielle des monstruosités" cosi si esprime: Depuis GEOFFroy SAINT-HILAIRE, quelques physiologistes ont signalé le fait de la production des monstres dans l'incubation artificielle. Prevost e Dumas parlent de ces faits dans leur célèbre travail sur le développement du poulet qu'ils publièrent en 1828 , mais en se bornant à une simple indication. Les résultats qu'ils ont obtenus sont, par conséquent, perdus pour la science. Si sa che sperimentarono l'elettricità, ma non hanno dato nessun dettaglio sui loro procedimenti. 
e Quajat (9) intorno all' influenza dell' elettricità (strofinamento, fioceo elettrico etc. etc.) sul precoce dischiudimento delle nova del Bombix mori, poichè molto indirettamente mi riguardano, vengo senza altro ai lavori di $W$. Roux. Riassumere per esteso le sue interesssanti ricerche sarebbe a dir vero uno dei compiti più ardui che mai si possano immaginare. La messe larghissima di osservazioni e le descrizioni oltre ogni credere minnte degli innumerevoli fatti osservati, mal si prestano ad essere con esattezza riassunte. Per conseguenza mi limito a riferire qui le parti che sono in più stretta relazione col tema da me preso a svolgere. Roux si è occupato di studiare l'azione di correnti elettriche di breve durata sulla prima divisione delle uova di Anfibi e sulle modificazioni che sono capaci di indurre nell' aspetto e nella loro struttura. Oltre a ciò come materiale di studiosi ̀̀ servito pure della Lacerta agitis, Telestes Agassizii, del Gallus domesticus, dell' Aethalium septicum, dell' Hydra fusca e dei mammiferi.

In un primo lavoro (10) comparso nel 1889 .al capitolo intitolato "Wirkung der Elektrieität auf die Richtung der Zelltheilung" si trova la seguente conclusione: »Eier, welche sofort nach der Befruchtung in eine relativ weite Glasröhre aspirirt und zwischen die Pole des Elektromagneten gelegt worden waren, ließen gleich den von demselben Strom direkt umströmten Eiern keine bestimmte Einstellung: ihrer ersten Furche erkennen."

In un secondo lavoro dato alla luce nel 1891 (11) l'autore dice di avere esperimentato sulle nova di Anfibi (Rana fusca e Triton alpestris) l'azione di correnti indotte e costanti e cosi conclude riguardo alle prime: „Der Wechselstrom vermag somit als solcher weder bei Durchströmung noch bei Umströmung der Eier eine richtende Wirkung auf die Theilung auszuiiben«. Altrettanto sarebbe secondo Roux per le correnti costanti. Infatti cosi si esprime riguardo a esse: „Damit ist dargethan, dass auch der Gleichstrom als solcher auf die Richtung der ersten Theilung des Furchungskernes und des Eileibes eine bestimmeude Wirkung nicht auszuüben vermag". Ma ciò che colpisce sopratutto l'attenzione sono le particolarità che si osservano alla superficie di uova sottoposte subito dopo la loro fecondazione all' azione di correnti elettriche di brevissima durata. Esse si possono cosi riassumere: comparsa in direzione degli elettrodi di due campi polari separati da un' anello equatoriale, solchi più $o$ meno profondi ai loro limiti quando abbiano agito forti correnti, addensamenti e disposizioni speciali del pigmento e formazione di 
aree chiare nel territorio dei campi polari. Liinsieme di questi fenomeni che sogliono verificarsi appunto nelle nova le quali non presentano ancora traccia di segmentazione chiama l'autore »Morphologische Polarisation«. Se invece si sottopongono all' azione dell'elettricità nova allo stadio di blastula e gastrula, quando sieno normali, ogni cellula mostra a se due campi polari e un' equatore. Si ha allora la cosi detta "Specialpolarisation der Zellen". Quando le uova sieno indebolite con un riscaldamento a $40^{\circ} 0$ col raffreddamento per mezzo del ghiaccio o con un debole avvelenamento per mezzo di una soluzione carbolica, si ha dapprima una iniziale e temporanea "Spezialpolarisation", poi una "Generalpolarisation" con due grandi campi polari ed un grosso anello equatoriale; cosi che la blastula e la gastrula indebolite si comportano come l'uovo insegmentato. L'uova uccise da una temperatura di $48^{\circ}$ C. o avrelenate con acido carbolico non reagiscono più. Questa reazione elettrica, si manifesta fino a un certo punto anche in organi dell'embrione. Gli organi dell' adulto non reagiscono in maniera più apprezzabile con formazione di campi polari e anelli equatoriali tanto alle correnti costanti che galvaniche. Oltre a ciò Roux eletrizzando nova segmentate ha osservato la fuoriuscita di materiali che egli chiama "Extraovate "Oltre però un determinato stadio di segmentazione questi particolari "Extraovate " non si formano più; ma si ottengono più facilmente intiere cellule isolate la cui maniera di comportarsi rispetto all' elettricità è del più alto interesse. Queste sieno brune o bianche non danno luogo (bei Durchströmung) abitualmente a campi polari, ma ad ambedue i poli scoppiano, si vuotano completamente del contenuto e se non esistono ostacoli esterni ciò accade nella direzione della corrente. $\mathrm{Si}$ ha allora una speciale formazione costituita come da due coni riuniti per gli apici. Nel punto di riunione la membrana costituisce una piccola massa di non più che $1 / 100$ dell' intiero volume cellulare. Constatati questi fatti Roux ricorda le ricerche di KüHNe e Verworn che io qui brevemente riferisco non solo perchè hanno una stretta relazione con le osservazioni di Roux ma anche perchè esse potranno servirmi a spiegare alcuni dei fatti da me osservati. KüHNE (12) ha condotto i suoi esperimenti sui Protisti e ha redato che questi per l'azione di correnti elettriche si fanno rilevati in corrispondenza dei poli e presentano segni di probabile distruzione. Eccitando un' ameda con una corrente costante la parte rivolta all' anode diviene oscura e si trasforma in una sostanza avente l'aspetto dei granuli di sago; la 
parte rivolta al catode mostra al contrario nel suo interno e sulla sua superficie numerose formazioni vescicolari. Se si fa agire sull' Actinosphaerium Eichornii una debole corrente indotta, i prolungamenti rivolti verso gli elettrodi si ritirano, mentre che quelli originati lateralmente all' animale e orientati rettangolarmente alla direzione della corrente rimangono immutati. Scoppiano pure soltanto sulle parti polari le vescicole della sostanza corticale. Adoperando correnti costanti, la parte rivolta verso l'anode si liquefà, si ridnce in una poltiglia, mentre la parte diretta verso il catode rimane inalterata. Nelle rapide chiusure di corrente come pure nelle interruzioni, si verifica in queste parti retrazione di pseudopodi e scoppio di vescicole. Qualora le correnti elettriche sieno molto intense questi fenomeni, si osservano su tutta la superficie dell' animale. Tali fenomeni, quali retrazione di pseudopodi, scoppio di vescicole, e disgregamento della porzione rivolta verso l'anode spiega KüHNe come effetto di contrazione in eui il protoplasma di questi protisti entrò per opera dello stimolo elettrico. Verworn (13) conferma nella intiera serie di Protisti le ricerche di KüHNe. Dice inoltre come anche alcuni flagellati, ciliati e bacteri presentano sulla parte rivolta verso l'anode il cosi detto eccitamento di chiusura, mentre nell' impiego di correnti indotte, l'A ha osservato come KüHNE su ambedue le parti polari un disfacimento granulare. In un' altra serie di ricerche (14) VERWorN ha potuto stabilire che nell' Amoeba limax, verrucosa e diffluens trattate con correnti galvaniche, il protoplasma ialino si raccoglie al catode, mentre il protoplasma granulare all' anode e nella Pelomyxa patustris resti del protoplasma ialino al catode si dispongono in formazioni vescicolari che fanno protuberanza. Roux ha creduto di mettere in relazione i risultati di KüHNE e VERWORN coi suoi e fa osservare come anche la sostanza embrionale dei vertebrati ha facile tendenza a distruggersi, mentre non è più cosi nel vertebrato adulto e conclude che $\gg$ Die lebende Zellleibsubstanz der frühen Entwickelungstadien der Wirbelthiere besitzt eine starke, zum Theil eigenartige Reaktionsfähigkeit auf elektrische Einwirkungen, anf Gleichstrom, Wechselstrom, und auf die Sehläge der Leydener Flasche, welche den Zellen des älteren Thieres nicht mehr zakommt, welche aber bei den Protisten und bei Cölenteraten (Hydra) während des ganzen Lebens sich findet. Q Quale significato poi abbiano certe apparenze dovute alla cosi detta localizzazione polare, le quali si verificano nelle uova, negli embrioni o in alcuni organi embrionali, possiamo subito dire ehe esse, come le dimostrano anche $i$ 
fatti osservati nelle esperienze eseguite sui Protisti, celenterati etc., non stanno a rappresentare altro che delle vere e proprie alterazioni. E Roux cosi si esprime in proposito: „Bei unseren lebenden Objekten ist wohl zu vermuthen, dass mit der sichtbaren ,morphologischen Polarisation eine elektrische Polarisation im Sinne der Physiologen verbunden ist, ganz abgesehen von der sogenannten, inneren Polarisation feuchter Leiter" nach Du BoIs-REYmond; verhält sich doch bei den Muskeln und Nerven nach L. Hermans jeder absterbende Querschnitt negativ gegen den lebenden; und nnsere Polfelder sind, wie wir an den Froscheiern sehen, eine Substanz, welche an den Theilungsvorgängen der Zelle nicht mehr Theil nimmt, sondern unter Umständen von den Zellen direkt abgestoßen, eliminirt wird, und welche auch nicht mehr jener Veränderungen (Vacuolisation etc.) fähig ist, wie sie sonst beim allmählichen Absterben der Eier beobachtet werden. "

Con le osservazioni di Roux termina la serie delle ricerche sperimentali intorno all' influenza dell' elettricità sullo sviluppo delle uova dei vertebrati. Prima peró di chiudere questo capitolo credo utile dare un cenno di altri lavori riguardanti gli effetti di stimoli elettrici su elementi cellulari appartenenti a tessuti e organi adulti, perchè essi al pari di quelli di KüHNe e VERworn già citati, mi saranno di non poco aiuto alla interpetrazione di certi fenomeni.

Hermann (23) sperimentando sulle larve di Rana e sugli embrioni di Pesci vide che se si faceva passare una corrente attraverso ai vasi nei quali essi si trovavano, si disponevano alla chiusura della corrente in modo da avere la testa rivolta verso l'anode e la coda verso il catode.

VELtex (15) studiando l'azione dell' elettricità sui movimenti del protoplasma ha veduto che correnti indotte molto forti fatte agire su aggregati di cellule o su cellule isolate determinano moti rotatori nel loro contenuto. La rotazione elettrica ha la più grande somiglianza con la rotazione vitale; ambedue obbediscono alla medesima legge. Correnti indotte 0 costanti inducono nei grauli più grossi inclusi nelle cellule e in altre particelle, movimenti di rotazione sul proprio asse perfettamente analoghi a quelli potuti osservare durante la vita nei granuli di Clorofilla di alcune cellule vegetali. Esagerandosi i moti rotatori, i granuli possono fuoriuscire dalla cellula.

Feld (16) esaminando il sangue di un giustiziato con la corrente elettrica 5 minuti dopo la morte, ha potato constatare nei corpuscoli 
l'esistenza di un numero maggiore 0 minore di granulazioni e protuberanze costituite di protoplasma. Conclude Fell che secondo l'intensità o la durata della corrente elettrica si puó avere vitalità esagerata, elettrolisi vera e propria, diminuita e spenta vitalità del protoplasma.

Dineur (17) studiando con speciali apparecchi la sensibilità dei leucociti all' elettricità, ha veduto che $\mathrm{i}$ leucociti si portano in gran numero al polo positivo e provvisti di pseudopodi presentano una manifesta vitalità, mentre quei pochi che si trovano al polo negativo sembrano morti 0 dotati di minima vitalità. Negli esperimenti di controllo si è dato cura di eliminare tutte le possibili cause di errore quali la sensibilità tattile, il chemiotaxismo, la cataforesi sviluppata dalla corrente. A proposito di quest' ultima dice: „Nous savons en effet que des particules inertes tenues en suspension dans un liquide, que traverse un courant, son charriées par ce dernier dans sa direction, c'est-a-dire du positif au négatif".

Pieraldini (24) studia le anomalie del processo cariocinetico provocate sperimentalmente con l'elettricità. L'A. cosi conclùde: "La corrente galvanica non ha influenza diretta sulla cariocinesi; ma indirettamente tende ad annientarla, procurando metamorfosi regressive che possono giungere fino alla necrobiosi del tessuto su cui la corrente agisce. La corrente faradica convenientemente applicata ha un' azione piuttosto benefica per la riproduzione dell' epitelio epidermoidale, stimolando la divisione diretta delle cellule. Tali correnti indotte alternanti impediscono probabilmente il potere di orientazione del protoplasma necessario alla cariocinesi e per questo lo stimolo alla riproduzione si estrinseca con la divisione diretta. Una corrente interrotta che agisca in una sola direzione con intervalli brevissimi, produce uno stato di contrazione continua del protoplasma. Da ciò possono derivare figure cariocinetiche speciali che non hanno però carattere patologico.

$\grave{\mathrm{E}}$ in seguito ai risultati resi noti da alcuni degli autori che sono venuto fin qui enumerando e alle preziose osservazioni praticate in questi ultimi anni da tanti scienziati quali Roux, Her'rwig, Schultze, Morgan, Drtesch e altri che sorse in me il desiderio di ricercare l'influenza esercitata da correnti elettriche costanti sulle nova fecondate degli Anfibi. E mi sono accinto alle ricerche col proposito di studiare non solo le apparenze esteriori, ma di indagare eziandio le modificazioni di strattura e di definire il modo di azione dello stimolo da me impiegato. 


\section{Materiale e Metodi di studio.}

Come materiale ho scelto le uova di Salamandrina perspicillata Savi, perche esse sia per il volume, sia per la netta differenziazione di colorito tra le due metà dell' wovo, sia per la mollezza e trasparenza degli involucri servono mirabilmente per certe ricerche e posso senza dubbio dichiararle superiori a quelle di quasi tutte le altre specie di Anfibi urodeli. Ho diviso gli esperimenti in due serie; nella prima ho provata l'azione di correnti continue sulle uova fecondate senza traccia di segmentazione e sui diversi stadi di segmentazione fino alla gastrula, uccidendo le uova stesse poco dopo cessato l'esperimento per studiarne le apparenze esterne e le modificazione di struttura; nella seconda ho lasciato che le uova collocate nell' apparecchio poco dopo l'emissione sviluppassero sotto l'azione prolungata della elettricità. La prima serie l'ho condotta a termine nella primavera dell' anno passato; la seconda nel mese di Marzo dell' anno in corso. L'apparecchio che mi ha servito e di cui do qui il disegno è costituito da un tubo di vetro del diametro di $\mathrm{mm} .23$ montato su di un supporto di legno; il fondo del tabo è fatto da una lamina di platino a cui è saldato un filo di rame; un' altra lamina di platino con annesso filo di rame pure saldato e di un diametro di poco minore di quello del tubo può essere tolta a piacere e adattata a una distanza maggiore 0 minore della precedente a seconda del bisogno, e a seconda della quantità di uova

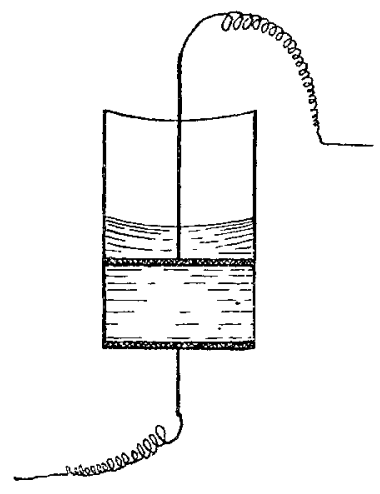
messe a sperimentare. Nella seconda serie di ricerche ho adoperato un tubo di vetro più largo e precisamente del diametro di mm. 40 . Uguali tubi senza alcun apparechio e chiusi semplicemente in fondo da un sughero mi hanno servito per le prove di controllo. Ho costante; mente tenuto conto della temperatura dell' ambiente, della quantità di acqua adoperata, delle diminazioni, inevitabili anche nelle più costanti sorgenti di elettricitá, verificatesi nel corso degli esperimenti. Mi sono servito di elementi a bisolfato di Mercurio alti mm. 60 e del diametro di mm. 48. Ho fatto agire correnti molto forti, di media intensità, deboli e debolissime. Dopo numerosi saggi ho potato stabilire che due dei detti elementi sviluppavano in generale una 
corrente di tale intensità da permettere la vita delle vova purchè non fosse sorpassato un determinato limite di tempo.

Un' apposito galvanometro mi segnava il grado di intensità della corrente che per una coppia mi si presentò costantemente data da circa A. 0,000507 . L'esperimento non durò nella $1^{\text {a }}$ serie mai più di 6 ore. Quando si prolungava oltre questo periodo come nella $2^{\text {a }}$ serie, l'acqua reniva cambiata nei tubi; anzi a questo proposito devo dire che disposi un semplice apparecchio per cui nelle ultime esperienze della seconda serie l'acqua veniva continuamente rinnovata. Cessata l'esperienza, il materiale si lasciava a se generalmente per circa due ore; veniva quindi, dopo essere stati tolti gli involucri piu esterni con tutta la delicatezza, fissato in soluzione satura acquosa di sublimato ove rimaneva per un tempo variabile da $1 / 2$ a 1 ora; lo passavo quindi in alcool al 50\% iodato, indi in alcool a 60, 70, e finalmente in quello a $85 \%$.

Per ottenere serie il più possibilmente perfette quali c̀ concesso ottenere da nova ricche in dentoplasma e fissate in sublimato, le tenevo 9 ore circa in toluolo e 24 ore in paraffina alla temperatura mai superiore ai $53^{\circ} \mathrm{C}$. Le sezioni praticate con un microtomo Thoma-Becker secondo un piano meridiano sono state di uno spessore variabile, a seconda del bisogno, dai 10 ai $15-20 \mu$ e incollate sul coprioggetto con l'albumina glicerinata (MAJER). Per evitare anche il minimo disgregamento delle sezioni, cosa facile ad accadere anche nelle pit̀ perfette inclusioni, quando si è costretti a far passare le sezioni stesse per una serie numerosa di liquidi di varia densità, ho immaginato il seguente processo. Fissate le fette ai vapori dell'aequa bollente, tolta la paraffina eon toluolo e eliminato il toluolo con l'alcool assoluto, togliero da questo i vetrini e lasciavo che alla temperatura dell' ambiente ne evaporasse una certa quantita; poi stendevo sulle sezioni un sottile strato di collodione allungato (collodione p. 2, alcool assoluto p. 3, etere solforico p. 3) e quindi immergevo $i$ vetri con le sezioni in alcool a $70 \%$. Con questo mezzo semplicissimo si possono eséguire tutti i procedimenti senza che le fette risentano più il benchè minimo danno dai reagenti. Riguardo ai mezzi di tinzione anche questa volta, per la rapidità della colorazione, e per la sua nettezza ho dato la preferenza al l'Haemalaun di MaJer. La direzione della corrente fu in ambedue le serie dal polo regetativo a quello animale e non appena collocate le nova nel tubo di esperimento mi assicuravo sempre che esse fossero tutte orientate col p. animale all' insù. 


\section{Azione dell' elettricità sulle uova fecondate non segmentate.}

Apparenze esteriori. I risultati delle ricerche, in questa parte, sebbene non completamente e ciò credo doruto al fatto che il tempo di eletrizzazione fu nelle mie esperienze di gran lunga superiore, concordano con quelli di Roux. Uno dei primi mutamenti da me notato e che mi si è offerto sotto varie forme e gradi è relativo alla distribuzione del pigmento. Le fig. 19, 20 rappresentano due aova nelle quali il segmento nero appariva notevolmente scolorito; il pigmento trovavasi raccolto in ammassi più o meno regolari presentanti delle aree intensamente scure. Questi ammassi erano circondati da solchi assai appariscenti nelle sezioni (fig. 52a, 52b, 53a, 53b). La fig. 21 mostra un uovo avente al polo animale quasi completamente scolorato, un soleo a forma di lettera $\mathrm{C}$ e il pigmento in tenue quantità raccolto in corrispondenza dei labbri di detto solco. Invece l'uovo rappresentato nella fig. 18 aveva un piccolo ammasso di pigmento e accanto un' area allungata chiara con un punto nero circondata da un solco abbastanza profondo come si può vedere dalle fig: $50 a, 50 b$. In altre uova che non ho riprodotte ho notato nel polo animale aree biancastre varie nel volume e nella quantità, rilevate (extraovate di Roux). Il massimo grado di fuoriuscita di materiali vitellini è rappresentato nella fig. 22 ove si vede l'uovo diviso in due porzioni da un soleo abbastanza profondo ma incompleto; la porzione superiore è esclusivamente composta di sostanza fuoriuscita; nella porzione inferiore è rimasta abbastanza regolare la disposizione nei materiali costitutivi dell' uovo. Ciò che si rileva dallo studio delle sezioni di quest' novo corrisponde perfettamente alle apparenze esteriori. Le particolarità che son venuto brevemente riassumendo si verificano quando la corrente elettrica non sia fatta agire oltre un certo periodo di tempo, e non abbia una intensità maggiore di quella da me più sopra indicata; che se si prolunga la durata dell esperimento o si aumenta l'intensità dello stimolo intervengono alterazioni tali che l'uovo è condotto ben presto a morire e allora esso ci si presenta come una massa sferica di un colorito uniforme bianco opaco. Sezionando queste ultime uova e. sottoponendole all' esame microscopico, si rileva un grado maggiore o minore di liquefazione del protoplasma e la scomparsa totale dei nuclei.

Struttura - Protoplasma. Assai interessante è lo studio microscopico. Riassumo i fatti che è dato ricavarne. In tutte le uova 
indistintamente si nota un disordine nella distribuzione dei materiali costitutivi. Fatta astrazione dalla disposizione che assume il pigmento, poichè essa può venire esattamente apprezzata dalla semplice osservazione delle apparenze esteriori, si vede come il deutoplasma raccolto in prevalenza al polo vegetativo tenda a portarsi verso il polo animale. In alcune nova ho potuto osservare delle vere e proprie masse allungate a forma di colonne disposte parallelamente che avevano invaso la parte destinata ad accogliere il protoplasma formativo il quale costituiva come degli isolotti, situati tra una colonna e l'altra. Altre volte tali correnti date da questi ammassi allungati diretti verso il polo animale invece di essere in numero variabile a seconda dei casi, erano rappresentate da una sola colonna molto voluminosa. Frequenti nel polo vegetativo zone più o meno estese costituite da un reticolo, lungo i filamenti e nelle maglie del quale trovansi abbondanti e fini granuli protoplasmatici; mentre scarseggia notevolmente il deutoplasma. Quando lo stimolo elettrico abbia agito per un tempo molto lungo anche la disposizione fin qui descritta si disordina e allora i materiali costitutivi dell' novo 0 si trovano mescolati intimamente fra di loro o si addensano in un punto qualunque della periferia nel polo animale e di qui, si fanno strada all' esterno. Contemporaneamente a questa fuoriuscita si osservano aree reticolate coi caratteri già detti spesso molto estese, non solo nel segmento inferiore dell' uovo ma anche nel superiore.

Nuclei. Tanto i pronuclei come i nuclei spermatici accessori che dirò subito si trovano con una certa costanza nelle uova normali di Salamandrina in numero variabile da poter dire trattarsi di vera e propria polispermia fisiologica, se la durata di eletrizzazione restỏ entro i limiti dovuti, non presentano notevoli modificazioni. In generale la loro sostanza cromatica si tinge meno intensamente che in condizioni normali. In qualche cąso essa non costituiva un reticolo ma era rappresentata da nomerosi e minuti granuli raccolti alla faccia profonda della membrana nucleare. Alcuni di questi granuli si trovavano al di fuori sparsi nell' area dal nucleo occupata. In qualche caso ho potuto vedere la cromatina raccolta in un ammasso irregolare, fortemente tinto dall' Haemalaun, contenuto in una sostanza finamente granulosa di colorito giallastro e con limiti poco netti. Riguardo alla resistenza che offrono allo stimolo elettrico i nuclei di varia natura che si trovano nell' uovo fecondato, devo dire che essa è minore per parte dei nuclei spermatici accessori. Mentre ho 
quasi sempre riscontrato poco modificati nella loro struttura e occupanti una posizione presso che normale $i$ due pronuclei, spesso non mi fu dato di rilevare traccia dei nuclei spermatici accessori.

\section{Azione dell' elettricità sulla segmentazione.}

Apparenze esteriori. Che nello sviluppo normale delle nova degli Anfibi si possano verificare deviazioni più o meno notevoli nella direzione dei piani di segmentazione, è stato già veduto. Pure nella Salamandrina, come sarà dimostrato da ricerche praticate nel nostro Istituto Anatomico, si verificano queste accidentalità. $\dot{\mathrm{E}}$ naturale che ciò io abbia tenuto in gran conto e difatti nelle descrizioni che sono per dare e nei disegni, io ho preso solo in considerazione quelle forme e quelle particolarità che mi si offrivano ripetutamente deviate in maniera più o meno considerevole dalla norma. Un fatto costante che richiamò subito la mia attenzione e che riferisco prima di ogni altro è dato dalla straordinaria profondità dei solchi e dalla rilevatezza eccessiva dei blastomeri. Se per »erste Theilung « nel senso di Roux noi dobbiamo intendere solamente il primo solco di segmentazione, allora io convengo con questo antore ehe sieno gli stimoli elettrici di breve o di lunga dürata, la direzione di esso viene di poco modificatà. Tuttavia a questo proposito faccio notare come non raramente il primo solco di segmentazione completo si presenti deviato in modo che l'uovo risulta diviso in due porzioni disuguali (fig. 1, 2). Quando cio avviene la superficie dell' uovo non è regolare e si notano delle protuberanze, più o meno rilevate nel polo animale in uno o in tutti e due $i$ segmenti nei quali l'uovo è diviso (fig. 1). Se però per »erste Theilung " dobbiamo intendere l'insieme dei primi solchi di segmentazione allora devo dire che non mi sarà difficile dimostrare come la divisione anche nelle prime sue fasi sia grandemente influenzata dall' azione dell'elettricità. Le fig. 12, 13, 15, 16, 17 rappresentano wova nelle quali la formazione dei primi solehi arvenne sotto l'influenza della corrente elettrica. Per quello che riguarda le fig. 12, 13, sebbene non dividenti in 4 uguali porzioni le uova, pure i due primi meridiani erano completi; fino a questo momento per conseguenza leggere possono considerarsi le deviazioni verificatesi nella divisione. Il disordine maggiore ha cominciato a originarsi con la comparsa del primo solco equatoriale e coi successivi meridiani. L'aspetto che presentano le uova delle quali è stato riprodotto quasi completamente di prospetto 
il polo animale, fa supporre che il $1^{\circ}$ solco equatoriale non sia stato ne completo ne regolare nella direzione. Che sia stato incompleto lo dicono i voluminosi blastomeri che vanno da un polo all' altro dell' novo in parte costituiti dalla porzione scura, in parte della chiara. Quale sia stato il meccanismo che ha dato origine alle deviazioni degli altri solchi è difficile il poterlo ricavare dalla straordinaria irregolarità di posizione, di forma e di volume dei blastomeri. Anche nell' novo riprodotto nella fig. 16 il primo equatoriale sebbene completo, è irregolare nell' andamento e uno dei due meridiani è incompleto dimodochè al disotto del solco equatoriale l'novo risulta diviso in 3 segmenti disuguali anzichè in quattro. Quest' ultimo fatto si esagera nelle fig. 15 e 17 . Nell' uovo della fig. 15 del quale è stato riprodotto di prospetto il polo animale, facendo astrazione dal $1^{\circ}$ solco equatoriale che è completo, si vede come i primi due meridiani si sieno limitati a dividere in quattro segmenti soltanto una piccola porzione del polo animale; è soltanto in questa poco estesa zona che sembra abbiano avuto luogo i primi fenomeni della divisione. Naturalmente il rimanente dell'uovo appariva indiviso. Anche più strano e interessante è ciò che si osserva nella fig. 17. Quest' uovo presentava, a differenza degli altri, distribuzione notevolmente irregolare del pigmento; esso si era agglomerato quasi in totaliti in un' area assai ristretta del polo animale; il resto dell uovo si presentava scolorito. Il primo soleo equatoriale era incompleto e $\mathrm{i}$ suoi estremi andavano a unirsi con quelli di uno dei meridiani il quale, pure incompleto, divideva soltanto una porzione del polo animale. Dal solco equatoriale decorrevano poi verso il meridiano 2 altri solchi; uno, che per la posizione poteva considerarsi come il secondo meridiano, incrociava nel punto centrale del polo animale il primo meridiano descritto, e si prolungava di poco al dila, dividendosi ben presto in due branche; l'altro brevissimo e parallelo a questo aveva una posizione più laterale. Dalla disposizione di questi solchi ne risultava che le due metà del polo animale erano divise ciascheduna in tre blastomeri, ma differenti tra loro per la forma e per l'aspetto. Del resto uno sguardo alla figura varrà meglio che qualunque descrizione per comprendere la singolarità e la anormalità profonda delle cose riferite. Non meno manifeste ed evidenti sono le particolarita che si verificano in stadi più avanzati di segmentazione e che sono rappresentati nelle fig. $3,4 a, 4 b, 5,6,7,8,9,10,11,14$. In tutte queste uova i fatti osservati si possono riassumere in una considerevole 
disuguaglianza nel volume dei blastomeri poichè accanto a blastomeri molto voluminosi se ne trovano altri assai meno grandi, altri di un' estrema piccolezza. Questi blastomeri cosi differenti per volume possono essere mescolati irregolarmente fra di loro, oppure separati ed aggruppati in zone differenti. Cosi per esempio in alcune uova ho potuto vedere (fig. 8-14) una piccola porzione di polo animale divisa in pochi blastomeri ma grossi e rilevati mentre il rimanente del polo animale occupato da una straordinaria quantità di piccole cellule e cosi piccole e cosi poco rilevate da essere impossibile la loro riproduzione sia con la camera lncida, sia a occhio nudo. Oltre a ciò, anche in quelle uova messe a sperimentare già divise precisamente allo stadio di 16 e per conseguenza coi primi due meridiani esistenti e completi, ho notato la scomparsa più o meno completa di questi solchi. Nell novo rappresentato delle figure $4 a, 4 b$ si notava solamente un resto dei due meridiani dato da un breve solco esistente soltanto in una delle meta. Oltre poi alla notevole e costante profondità dei solchi e rilevatezza dei blastomeri che ho avuto più sopra occasione di riferire, in qualche aovo ho notato deformazioni di un grado maggiore o minore. Il maggior numero però conservavano integra la loro forma sferica. Sulle modificazioni delle apparenze esteriori nelle uova sottoposte allo esperimento in stadi molto aranzati di segmentazione poco ho da dire, poichè esse sono, astrazion fatta da possibili deformazioni, assai poco rilevabili. Noto soltanto in uova messe a sperimentare allo stadio di blastula avanzatissima (prossima formazione di blastoporo) la comparsa di solcature nel polo animale più o meno profonde dirette in vario senso senza però segni di ordinata disposizione. Non voglio poi tacere ciò che concerne la resistenza delle uova all' elettricità costante; essa aumenta con la segmentazione; più si presentano segmentate le nova e più sono capaci di resistere allo stimolo elettrico.

A questo punto io dovrei esporre i risultati dell' esame microscopico del materiale illustrato; credo però opportuno di dare prima un cenno di quanto osservai nella seconda serie di esperimenti e ciò per completare quello che si riferisce alle apparenze esteriori presentate dalle uova che hanno subito l'influenza della corrente elettrica. Devo subito avvertire che prima di arrivare ai risultati ottenuti ho dovuto fare alcune esperienze di prova dalle quali ho ricavato che le uova non sopportano l'azione continuata di correnti costanti anche debolissime malgrado il rinnuovamento continuo dell' aequa; cosi non sopportano deboli correnti costanti quando i 
periodi di esperimento sieno numerosi e continuati per molte ore, anche se l'acqua vengo di continuo rinnovata e gli intervalli di riposo sieno di lunga durata. Le uova in questi casi finiscono col morire. Quando la morte si verifica dopo un certo tempo, le nova presentano sempre, fino al momento in cui è possibile rilevarle, anomalie di segmentazione aventi gli stessi caratteri di quelli da me più sopra descritti. L'esperienza che mi dette il più notevole risultato fu la $7^{\text {a }}$ in data del 26 Marzo. Nel tubo di esperimento furono messe 65 uova segmentate agli stadi di 2, 4, 8. „La distanza tra le due lamine di platino fu di $60 \mathrm{~mm}$ gli elementi adoperati 2. Alle $11 \frac{1}{2}$ si comincio l'esperimento; l'acqua reniva continuamente sostituita da quella che sgocciolava da un' ampia bottiglia spesso agitata e aereata. L'apparecchio funzionò regolarmente fino al mattino di poi a ore $9 \frac{1}{2}$. L'intensità della corrente era diminuita di poco più di $1 / 3$. A detta ora cessai l'esperimento ed esaminai ad una ad una le uova. Una ventina che oltre un' opacamento del l'involucro interno presentavano segni più o meno manifesti di morte furono gettate via. Le altre erano talmente progredite nella loro segmentazione che nulla di notevole potevasi rilevare dall esame della superficie. Messe a sviluppare in un' altro tubo di uguale diametro il giorno appresso (28 Marzo) nelle ore pomeridiane mostrarono molte (circa 30 ), più o meno equatorialmente situata, una fessura poco estesa ma assai profonda che, sebbene molto precocemente comparsa, non poteva avere per me altro significato che quello di un blastoporo. Il giorno di poi la fessura si estese notevolmente con direzione circolare e da questa estensione si originarono tappi vitellini generalmente molto ampi, limitati da solchi completi in quelle nova in cui l'invaginazione avvenne al disotto dell' equatore e incompleti in quelli in cui l'invaginazione stessa erasi formata proprio a livello dell' equatore o poco al disopra (fig. 22, 23). Il 3 aprile si iniziarono i primi mutamenti indicanti la formazione della doccia midollare. II di 5 in qualche uovo cominciavano a farsi rilevati i labbri della doccia, e fu soltanto nei giorni 8 e 9 che $\mathrm{i}$ labbri della doccia stessa accennavano a saldarsi. Non tutte queste nova aventi il tappo vitellino molto ampio poterono percorrere detti stadi di sviluppo, poichè alcune morirono prima che vi apparissero accenni di doccia. Tutte però si presentarono anomale e le varie fasi si svolsero con la presenza di un tappo vitellino moltó ampio. I disegni che io do di queste uova (fig. 24, 25, 26, 27, 28) sono cosi fedelmente netti e chiari che mi risparmio qualunque descrizione potendosi rilevare con 
tutta facilità a prima vista le anomalie in esse esistenti. Oltre lo stadio rappresentato dalla figura $28 \mathrm{non} \mathrm{mi} \mathrm{è} \mathrm{riuscito} \mathrm{condurre} \mathrm{le}$ uova. Il giorno 11 Aprile raccolsi morto l'ultimo uovo. Le altre 15 morirono tutte con segni manifesti di idropisia avanti la comparsa del blastoporo. Delle 8 esperienze che costituiscono la seconda serie, questa è la più concludente, e ho creduto di prenderla in considerazione sia per il numero delle uova anomale ottenuto, sia perchè in tutte, tranne lievissime differenze ebbi un tipo unico e costante di anomalia.

Struttura delle uova segmentate. Presso che cento sono state le uova da me sezionate allo scopo di studiarvi le intime modificazioni di struttura. Alcune delle figure qui annesse si riferiscono a quelle già riprodotte nelle loro apparenze esteriori. Cosi le fig. 30, $31,32,33,34,35,38$ rappresentano altrettante sezioni mediane delle uova disegnate nelle fig. $1,2,8,9,3,14,11$. Le altre riferisconsi in parte ad uova non riprodotte nei caratteri esterni o riprodotte ma non contrassegnate per una involontaria interruzione nell'ordine sempre scrupolosamente osservato, come appunto è di quelle che nella spiegazione delle figure si dicono appartenere all'esperienza $10^{\text {a }}$. Ciò poco monta. Io richiamo soltanto l'attenzione sopra certi fatti, alcuni dei quali si trovano in perfetta correlazione con le apparenze esteriori. $\mathrm{E}$ questi fatti sono: $1^{\circ}$ notevole disuguaglianza nel volume dei blastomeri; $2^{\circ}$ loro rilevatezza; $3^{\circ}$ deformazioni più o meno marcate; $4^{\circ}$ apparente scarsa 0 mancante segmentazione del polo .vegetativo anche in stadi avanzati di segmentazione e anche quando le uova furono messe a sperimentare procedute assai nella loro divisione; $5^{\circ}$ andamento anomalo di tutti $\mathrm{i}$ solchi in generale. Le figure, le quali non rappresentano altro che i contorni delle sezioni presi con la camera lucida, indicano queste cose in maniera cosi evidente che non trovo ne atile ne opportuno, darne di ciascuna una speciale descrizione.

Protoplasma. Poco differenti da quelle già riferite, sono le modificazioni nella distribuzione dei materiali costitutivi, nelle uova la cui divisione accade sotto l'influenza dell' elettricità. Facilmente rilevabili nei primi stadi di segmentazione, di mano in mano che questa si fa più avanzata certi caratteri non si apprezzano più. Uno dei fatti che si è offerto al mio studio con una certa frequenza e costanza è dato dal trovarsi entro i blastomeri aree non molto estese formate di una sostanza apparentemente omogenea e debolmente tinta dall' Haemalaun. 
Nuclei. Sono molto frequenti e numerose nelle uova sperimentate, tanto nel polo vegetativo come nel polo animale, sia nelle prime fasi della segmentazione come nella blastula a più strati, certe forme che presentano i seguenti caratteri: Sono aggregati di nuclei variabili per forma o volume, provvisti di membrana e di un contenuto; in questo scarseggia la cromatina; al contrario la membrana si tinge intensamente e forma un contorno scuro pinttosto spesso tantochè osservati con piccoli ingrandimenti hanno l'aspetto di vescicole. La forma alle volte è rotondeggiante, altre allungata; i contorni sono generalmente regolari. Se esistessero legami tra $i$ diversi frammenti nucleari non posso dire, poichè di regola si sono afferti al mio strdio sempre strettamente addossati l'uno all' altro. A causa di questa disposizione non ho potuto ptre stabilire la presenza di microcentri e relative irradiazioni. Il numero dei frammenti nucleari è vario; ne ho contati da 3,4 fino a 7,8 . Oltre a ciò non raramente nei blastomeri delle uova sperimentate mi sono imbattuto in speciali alterazioni dei nuclei. In alcuni casi il nucleo diventa un pó più voluminoso; i suoi contorni si fanno dentellati, il contenuto nucleare assume una tinta molto intensa tantochè è solo con l'aiuto di forti ingrandimenti che si può distinguere in esso un reticolo $\mathrm{i}$ cui filamenti sono disposti con un certo disordine e una sostanza apparentemente omogenea. Altre volte si vedono aree di sostanza finamente granulosa, di colorito grigiastro in cui o disordinatamente 0 con disposizione raggiata si trovano disseminati dei corti hastoncelli di sostanza cromatica. In altri casi invece la cromatina si trova raccolta in ammassi irregolari sempre intensamente tinti. Anche nei nuclei in scissione non è raro il caso di imbattersi in speciali alterazioni; rimane allora colpita tanto la parte cromatica quanto l'acromatica; i filamenti di quest' ultima si fanno meno distinti e possono o condensarsi o allontanarsi l'uno dell' altro. Fenomeni di cariolisi nel senso di Flemming o di Hermans non mi fu dato mai di rilevarne. Finalmente nuclei in riposo si possono vedere estremamente voluminosi con segni più o meno manifesti di rigonfiamento o frammentati in numerose e minute rescicolette apparentemente prive di cromatina contenute in una sostanza finamente granulosa, incolora, con un numero maggiore o minore di granuli di pigmento.

Modificazioni nella orientazione delle figure cariocinetiche. Sebbene il numero delle uova da me sezionate e studiate sia stato rilevante pure pochi sono i casi nei quali ho potuto sorprendere i nuclei in divisione. In stadi avanzati di segmentazione non ho 
riscontrato costanza nella direzione delle figure; ma sibbene un certo disordine, in correlazione con le disuguaglianze di volume, di forma e di posizione dei blastomeri. Notevole l'osservazione ehe riguarda l'uovo rappresentato dalle fig. 1 e 30 . Quest' novo diviso in due blastomeri disuguali, venne come tatti gli altri sezionato secondo un piano perpendicolare al piano equatoriale ossia ad angolo retto col meridiano già formato. Per conseguenza le figure cariocinetiche dei due respettivi nuclei che si trovavano allo stadio nel quale le anse sorelle si portano verso i poli, dovevano essere orientate in modo da trovarsi parallele al piano equatoriale per dar luogo al $2^{\circ}$ meridiano. Esse invece occupavano una posizione tale da essere a questo piano perpendicolari, parallele cioè al soleo di segmentazione.

Esposti i risultati delle mie ricerche mi vien fatto di rivolgermi le seguenti domande:

$1^{\circ}$ E possibile che uno stimolo elettrico di una determinata intensità e di una certa durata possa dar luogo ai fenomeni da me rilevati? E con quale meccanismo?

$2^{\circ}$ Le modificazioni che nno stimolo elettrico è capace di indurre nello sviluppo sono proprie e speciali di detto stimolo?

Le ricerche eseguite su organismi inferiori interi o su cellule isolate o aggruppate appartenenti ad organismi superiori, hanno dimostrato come le correnti elettriche possono determinare, insieme a varie altre modificazioni, dei movimenti nei materiali che li costituiscono.: Essi a seconda dell' intensità della corrente elettrica, sono rappresentati o da semplici moti di rotazione, o da vere e proprie correnti con date direzioni; finalmente esagerandosi con l'aumento dello stimolo questi movimenti, si può avere fuoriuscita dall'organismo: intero o della cellula, di una parte dei materiali. In altri elementi poi (lencociti) vennero notati speciali stati di contrazione del protoplasma rivelati in particolar modo da movimenti ameboidi più rapidi e da attive formazioni di pseudopodi. Per conseguenza uno stimolo elettrico può agire su una cellula o su di un' aggregato di cellule in due modi: determinando cioè delle contrazioni e dei movimenti nelle parti che le compongono. Io stimo opportuno di mettere in relazione questi reperti coi miei, e credo logica la supposizione, che quanto ho osservato nelle nova non segmentate cioè solchi, disordine nei materiali costitutivi dell'novo, loro fuorinscita e trasporto di essi da un segmento all' altro 
non siano altro che l'effetto di contrazioni, e di correnti determinatesi nell' uovo sotto l'influenza della corrente elettrica. Data l'esistenza di un tale disordine nella distribuzione dei materiali costitativi, non è difficile comprendere come la segmentazione non abbia potuto accadere in maniera normale e perchè essa si sia mostrata costantemente e notevolmente alterata nella direzione dei suoi piani. Inteso cosi il meccanismo di axione esercitato sulle uova dallo stimolo elettrico, mi resta a spiegare qualche interessante particolarità già notata e in special modo la deficiente segmentazione del polo vegetativo e l'esistenza in esso di quella zone reticolate scarseggrianti di deutoplasma. 'Ho detto più sopra che le correnti originatesi in una cellula $o$ in un organismo per opera di uno stimolo eletrico hanno determinate direzioni, vanno eioè nel senso della corrente; che materiali inerti i quali si trovino sospesi in un liquido, si portano dal polo positivo al negativo, e che nelle mie esperienze la corrente era diretta dal polo vegetativo al polo animale. Ora se pensiamo che tra i materiali costitutivi dell' uovo è da considerarsi come pressochè inerte, paragonato al protoplasma vero, il dentoplasma e che $\mathbf{i}$ suoi granuli godono di una certa libertà nell' interno dell' uovo, è naturale che essi sieno stati diretti in quantità maggiore o minore dallo stimolo elettrico verso il polo aminale. Tali correnti altre il trasportare materiali del polo vegetativo al polo animale e provocare quivi una orientazione differente nella distribuzione del protoplasma, devono avere certamente ostacolato l'emigrazione dei nuclei dal segmento superiore all' inferiore dell'novo, dando campo cosi alla segmentazione di svolgersi esclusivamente o quasi nel polo animale. Oltre a ciò dall' esersi una buona parte del deutoplasma portata verso il polo vegetativo, devono aver preso origine quelle zone reticolate nelle quali mentre si trovava in abbondanza tra le maglie e lungo i filamenti del reticolo il protoplasma, trovavansi al contrario in tenue quantita le sferule vitelline. Ma ho notato pure poli vegetativi apparentemente insegmentati anche in quelle nova messe a sperimentare coi primi meridiani completi. Mi fo ragione di questo fatto pensando come le correnti determinatesi entro l'uovo, sia per la loro intensità, sia per la loro durata derono avere facilmente distrutti i confini delle cellule già formate nel polo vegetativo e permesso ai materiali appartenenti a ciascuna di esse di mescolarsi tra di loro. Che sia cosi lo dimostra la fig. 54. L'uovo rappresentato fu messo a sperimentare allo stadio di 32 e venne adoperata una corrente piuttosto forte (3 elementi). 
Al termine dell' esperimento mostrava solo 6 blastomeri evidenti ma irregolari; tutto il rimanente della superficie si presentava di colorito irregolare, ma liscia e apparentementi insegmentata. In quanto ai nuclei polimorfi da me più sopra descritti devo dire che molti autori hanno rinvenute formazioni simili nelle uova normali di vertebrati durante la segmentazione. La maggiore frequenza peró nelle uova sperimentate e il fatto che in uno stesso novo se ne possono vedere in discreto numero anche nelle cellule del polo animale, non mi fa del tutto escludere che alla loro produzione cooperi in qualche maniera lo stimolo elettrico $^{1}$ ). Quale significato abbiano poi alcune delle forme di alterazione nucleare da me descritte e se esse sieno in relazione con lo stimolo adoperato io non mi sento di poterlo con sicurezza asserire. Braus (18) nel Triton alpestris ha fatto analoghe osservazioni. Questo autore non arrischia di affermarlo; ma fa intravedere l'idea che nuclei in preda a tali degenerazioni non sieno quelli derivati dal primo nucleo di segmentazione; ma :che si tratti invece di nuclei spermatici accessori i quali dopo avere più ó meno lungamente vissuto o sono morti o in via di morire. Riguardo poi al fatto se cioè le modificazioni prodotte della corrente elettrica sullo sviluppo sieno speciali e caratteristiche di questo stimolo, io, senża avere la pretesa di risolvere in modo assoluto la questione, rivolgo l'attenzione alle ricerche in questi ultimi tempi eseguite sulle uova degli Anfibi e noto analogie fra molte, anzi fra le prịcipali e più importanti anomalie studiate. Sieno dunque fisici o chimici gli stimoli adoperati, oppure senza che venga fatto uso di determinati stimoli, si trovino le uova in un mezzo non

1) Senza entrare nel merito della questione devo dire a questo proposito che Beldonor (Archives Italiennes de Biologie. $3^{m e}$ Année. 1884. Tome VI. pag. 52-5i) nelle uova normali di Axolotl ha veduto un fatto analogo. Ecco cosa conclude l'A. rispetto ad esso: $\gg$ Dunque nella segmentazione delle uova di $A x o l o t l$ (e forse anche di molti altri animali) la prima parte del ciclo cariocinetico presenta gli stessi fenomeni che si osservano nelle cellule dei tessuti. La seconda parte al contrario devia dal ciclo schematico di Flemming, ossia essa non ripete all' inverso i fenomeni precedenti. Effettivamente vicino ai poli del fuso prossimo a scindersi, i fili cromatici probabilmente rinforzati dalla acromatina, formano delle piccole cellule piene di succo nucleare che fondendosi tra di loro, rigenerano il nucleo. $\mathrm{Ma}$ a tale interessante e non ancora risolnta questione si collegano anche i nomi di BALFoUR, RÜCKJRT, KASTSCHENKo, HoFFMANN, VAY, TODARo, BRAUS, GRöN̦Roos e di altri. Una estesa bibliografia si trova nello recente memoria di GRöNRoos »Zur Entwickelungsgeschichte des Erdsalamanders». Anatomische Hefte (MERKel u. BonNeT). Heft XVIII (VI. Bd. Heft II). 1895. pag. 155. 
perfettamente corrispondente a quello in cui sogliono abitualmente sviluppare, il risultato finale è presso a poco identico. Per conseguenza mi sembra giusto ritenere col DARESTE che anche nello sviluppo degli Anfibi al pari che in quello degli Uccelli, l'elettricita agisce come tutte le altre cause che modificano l'evoluzione e imprimono ad essa una direzione differente dallo direzione normale. Ho creduto di limitarmi a queste sole e poche considerazioni di indole generale perchè le somiglianze esistenti nella più gran parte klelle anomalie di sviluppo delle uova degli Anfibi sperimentalmente provocate con mezzi differenti, l'elettricità compresa, mi dispensano di fare su ciascheduna forma anomale da me osservata certi paragoni e certe riflessioni. Inteso il meccanismo di azione dello stimolo elettrico, notate e descritte le modificazioni she questo è capace di indurre nell' novo prima e durante il processo di segmentazione, è facile capire in qual maniera abbiano avuto origine più tardi certe anomalie, perchè si sieno verificate deviazioni così profonde nelle ulteriori fasi dello sviluppo.

\section{Zusammenfassung,}

Ein konstanter elektrischer Strom von einer gewissen Dauer und bestimmter Richtung und Intensität vermag in den Bestandtheilen der Eier Bewegungen hervorzarufen. Diese Bewegungen fiihren verschiedene Modifikationen sowohl in der äußeren Erscheinung wie im inneren Ban der Eier herbei, welche sich folgendermaßen zusammenfassen lassen:

Unregelmäßige Vertheilung des Pigments, Anftreten (an den eben befruchteten Eiern von mehr oder weniger tiefen Furchungen am animalen Pole, mehr oder minder reichlicher Austritt von Eibestandtheilen, merkliche Abweichungen in der Richtung der Furchungsebenen, außergewöhnliches Hervortreten der Blastomeren, Ungleichheit derselben in Größe und Form, Übertragung von Bestandtheilen des vegetativen Poles zum animalen Pole, Bildung netzförmiger Zonen in einem oder in beiden Abschnitten des Eies je nach Stärke und Daner des Reizes, spärliche oder mangelnde Segmentirung des vegetativen Poles, unregelmäßiger Verlauf aller Furchen. Alle diese während der Furchung eingetretenen Verändernngen lassen ihre Wirkung auch an den späteren Entwickelungsstadien (Gastrula, Medullarrinne) wahrnebmen, welche tiefgreifende Unregelmäßigkeiten aufweisen (s. Fig. 23 bis 24).

Anßer auf das Eiprotoplasma wirkt der elektrische Reiz wahrscheinlich gleichfalls kräftig auf die ruhenden wie die sich theilenden Kerne, indem er Fragmentirungen, oder eine abweichende Orientirung derselben während der Theilung oder mehr oder weniger tiefgehende Veränderungen an denselben erzengt.

Vergleicht man die erhaltenen Resultate mit allen jenen Ergebnissen, welche mit verschiedenen Mitteln bei experimentellen Üntersuchmnen an Amphibieneiern gewonnen wurden, so lïsst sich mit ziemlicher Wabrscheinliehkeit 
annehmen, dass die durch einen elektrischen Reiz in der Entwickelung erzengten Anomalien diesem nicht speciell und ausschließlich eigen sind; sondern dass der elektrische Strom wirkt wie alle anderen Ursachen, welche die Entwickelung modificiren und ihr eine von der normalen abweichende Richtung anweisen.

\section{Letteratura.}

1. M. Ruscons, Über künstliche Befruchtungen von Fischen und über einige neue Versuche in Betreff künstlicher Befruchtung an Fröschen. Archiv f. Anat., Physiol. u. wissenschaftl. Medicin. Heransgeg. จ. JoH. MüLLer. 1840. (Citato da Prejer in: Physiologie spéciale de l'Embryon. Paris 1877. Trad. Wiet.)

2. L. Lombardinr, Forme organiche irregolari negli uccelli e nei batrachidi Pisa, Tip. Nistri. 1868.

3. G. Fasora, Azione di deboli correnti indotte sullo sviluppo delle ova di Rana. Archivio per le scienze mediche. Vol. 11. No.21. 1887. pag.,439-448

4. B. C. A. Windue, On certain early malformations of the Embryo. The Journal of Anatomy and Physiology normal and pathological. Vol. 27; New series Vol. 22. 1893 . pag. 436-453.

5. Prevost e Dumas, Mém. sur le dév. du Poulet dans l'œúf. Ann. de Sci. Nat. 'T. XII. (Série I.) pag. 417, (Citato da WindLe.)

b. B. C. A. Windue, On the effects of electricity and magnetism on development. Journal of Anatomy and Physiology ete. Vol. 29. 1895.

7. Dareste, Recherches sur l'influence de l'électricité sur l'évolution de l'embryon de la poulet. Note Comptes rendus hebdomadaires des séances de l'Académie des Sciences. T.121. No.25. 16. Dicembre 1895. pag.955-957.

8. VERSON e QUAJAT, Bollettino mensile di bachicoltura. 1874.

9. Beillati e QuaJat, Ibidem. Serie 20, Annata 120, No 9 e 10, Dicembre 1s91, e Atti. Isti. Veneto. Serie VII ${ }^{2}$. T. III ${ }^{\circ}$. pag. 1715-1736. 1892.

10. W. Roux, Über die Bestimmung der Hauptrichtungen des Froschembryo im Ei und über die erste, Theilung des Froscheies. Breslauer äratliche Zeitschrift. 1889. Nr. 6-9, vom' 27. März łan und Gesammelte Abhandlungen über Entwickelingsmechanik der Organismen. Leipzig 1895. Bd. II. pag. 277.

11. W. Roux, Beitrag VI zur Entwickelungsmechanik des Embryo. Über die morphologische Polarisation von Eiern und Embryonen durch den elektrischen Strom sowie über die Wirkung der ersten Theilungen des Eies. Sitzungsberichte der Kaiserlichen Akademie der Wissenschaften in Wien. Mathematisch-naturwissenschaftl. Klasse. Bd. CI. Abth. III. Vorgelegt in der Sitzung vom 17. December 1891, mit 3 Taf., und Gesammelte Abhandlungen etc., pag. 541, und Biologisches Centralblatt. 15. Bd. Nr. 10-15. Mai 1895. pag. 385-390.

12. W. KüHNE, Untersuchungen über das Protoplasma und die Kontraktilität. Leipzig 1864. (Citato da Roux.)

13. M. Verworn, Die polare Erregung der Protisten durch den galvanischen Strom. Pfü̈ger's Archiv f. Physiologie.. Bd. 45-46. (Citato da RouX.)

14. M. Verwora, Ibidem. Bd. 48. 
15. W. Velten, Die Einwirkung strömender Elektricität auf die Bewegung des Protoplasmas, auf den lebendigen und todten Zelleninhalt, sowie auf materielle Theilchen überhaupt. Sitzungsberichte d. Wiener Akad. Nr. 18.

16. FexL, The influence of electricity on Protoplasm. Am. Monthly Mic. Jl. Aug. 1890. (Citato da WrNole.)

17. E. Dineur, Note sur la sensibilité des leucocytes a l'electricité. Bulletin de la Société Belge de microscopie. Ann. 18. 1891-92. No.5. pag.113-118.

18. H. Braus, Über Zelltheilung and Wachstham des Tritoneies mit einem Anhang über Amitose und Polyspermie. Jenaische Zeitschrift. Bd. 29. Neue Folge 22. 1895. pag. 443-511.

19. M. Verworn, Allgemeine Physiologie. Ein Grundriss der Lehre vom Leben. Jena 1895.

20. Hermany, Einwirkung galvanischer Ströme anf Organismen. PfLÜGER's Archiv. Bd. 37. 1885. (Citato da VerworN.)

21. G. Pieralding, Anomalie del processo cariocinetico provocate sperimentalmente. Lo Sperimentale. Arehivio di Biologia. Anno L. Fascic. 1. Firenze 1896. pag. 32-70.

22. O. Hertwig, Urmund und Spina bifida. Archiv f. mikr. Anatomie. Bd. 39. 1892.

23. 0. Hertwig, Die Entwickelung des Froscheies unter dem Einfluss schwächerer und stärkerer Kochsalzlösungen. Ibidem. Bd. 44. 1895. Heft 3.

24. A. Gurwitsch, Über die formative Wirkung des veränderten chemischen Mediums auf die embryonale Entwickelung. Versuche am Frosch- und Krötenei. Archiv f. Entwickelungsmechanik der Organismen. Bd. III. Heft 2. 1896.

\section{Spiegazione delle figure tavola IX-XIV.}

Tutte le figure del No. 1 al No. 22 vennero disegnate con la camera lucida di Abbé, obiettivo 1 Hartnack, oculare 3, tubo chiuso, lunghezza del tubo mm 127. Le rimanenti, ad eccezione di quelle portanti i numeri $23,24,25,26$, $27,28,29$, con l'obiettivo No. 2.

Fig. 1-2. Uova messe a sperimentare poco tempo dopo la loro emissione senza traccia di segmentazione. Durata dell' eletrizzazione ore $\$ 1 / 2$.

Fig. 3. Uovo messo a sperimentare con i primi due solchi meridiani. Durata dell' eletrizzazione ore $41 / 2$.

Fig. $4 a, 4 b, 11$. Uova messe a sperimentare allo stadio di 16 blastomeri. Durata dell' eletrizzazione ore 4.

Fig. 5. Uovo messo a sperimentare con i primi due solchi meridiani. Durata dell' eletrizzazione ore 5 .

Fig. $6,7,9,10,12,13$. Uova messe a sperimentare con i primi due solchi meridiani. Durata dell' eletrizzazione ore 5.

Fig. 15, 16. Uova messe a sperimentare senzo traccia di segmentazione (poco dopo emesse). Durata dell eletrizzazione ore 5.

Fig. 8. Uovo messo a sperimentare allo stadio di 16 blastomeri. Durata dell'eletrizzazione ore 6 . La porzione tinta uniformemente in scuro appartiene 
al polo animale. Era costituita da numerosi e cosi piccoli blastomeri che non si sono potuti riprodurre.

Fig. 14. Uovo messo a sperimentare allo stadio di 16 blastomeri. Durata dell'eletrizzazione ore $4 \frac{1}{2}$. La porzione scura ha il significato di quella dell' uovo della fig. 8.

Fig. 17. Uovo messo a sperimentare senza tracia di segmentazione. Durata dell' eletrizzazione ore 6.

Fig. 18, 20, 21, 22. Uova messe a sperimentare poco dopo la emissione. Durata dell' eletrizzazione $1 / 2$ ora. Elementi 3. In 20 e 21 polo animale veduto di prospetto. In 18 si vede una piccola porzione del polo vegetativo. La linea curva a forma di $\mathrm{C}$ che si vede nella fig. 21 rappresenta un solco ai margini del quale si è andato a raccogliere quasi tutto il pigmento. Nella fig. 22 è rappresentato nella parte superiore dell' novo un voluminoso extraovate (Roux).

Fig. 19. Uovo messo a sperimentare poco dopo la sua emissione. Durata dell'eletrizzazione ore 6 . Un solo elemento venne adoperato.

Fig. 23. Esperienza $7^{a}$, serie $2^{a}$ ampio tappo vitellino (vedi testo).

Fig. 24. Esperienza 7a, serie $2^{\mathrm{a}}$ come sopra.

Fig. 25, 26, 27. Esperienza 7a, serie 2a. Tre docee midollari anomale. Coesistenza di ampio tappo vitellino.

Fig. 28. Esperienza $7^{\text {a }}$, serie 2a. Ravvicinamento dei labbri della doccia. Accenni di pieghe midollari sul labbro sinistro.

Fig. 29. Esperienza 7a, serie 2a. Doccia midollare chiusa. Accenni di pieghe midollari. Estremo cefalico rilevato, ampio tappo vitellino.

Fig. 30, 31, 32, 33, 34, 35, 38. Sezioni mediane delle uova rappresentate rispettivamente nelle figure $1,2,8,9,3,14,11$.

Fig. $36 a, 36 b, 36 c, 39,40 a, 40 b, 40 c, 43,44,45,46,49$. Esperienza 10a. Com $\Theta$ a fig. $6,7,9,10,12,13,15,16$.

Fig. 37, 42. Esperienza 9a. Come a fig. 5 .

Fig. 41, 47, 48. Esperienza 26a. Come a fig. 14.

Fig. 50 $a, 50 b$. . Sezione mediana dell' novo rappresentato nella fig. 18.

Fig. $51 a, 51 b$. Sezione mediana dell' uovo disegnato nella fig. 21.

Fig. $52 a, 52 b$. Sezione mediana dell' novo disegnato nella fig. 20.

Fig. $53 a, 53 b, 53 c$. Sezione mediana dell' uovo diseguato nella fig. 19.

Fig. 54 (tav. XIV). Uovo messo a sperimentare allo stadio di 32 blastomeri. Durata dell' eletrizzazione ore $41 / 2$. 